\section{Gaussian-like spectrum equalization using an all-fiber lattice filter with symmetric feedback structure}

\author{
Zhao Gang Dong \\ Nanyang Technological University \\ School of Electrical and Electronics \\ Engineering \\ Nanyang Avenue, Singapore 639798 \\ Ying Zhang, MEMBER SPIE \\ Singapore Institute of Manufacturing \\ Technology \\ 71 Nanyang Avenue, Singapore 638075

\section{Qi Jie Wang} \\ Yeng Chai Soh \\ Nanyang Technological University \\ School of Electrical and Electronics \\ Engineering \\ Nanyang Avenue, Singapore 639798
}

Abstract. We present an all-fiber filter with symmetric forward and feedback lattice structures. Using the proposed filter, Gaussian-like spectra can be equalized in the maximally flat sense while the output power is maximized. As an illustrative example, the proposed filter is designed to flatten the output spectrum of a superluminescence light-emitting Diode (SLED). Compared to existing filters, the proposed filter achieved better performance in experiments. () 2005 Society of Photo-Optical Instrumentation Engineers.

[DOI: 10.1117/1.1907188]

Subject terms: fiber optics; filters; spectrum equalization; broadband light sources.

Paper L040765 received Oct. 20, 2004; revised manuscript received Jan. 13, 2005; accepted for publication Feb. 16, 2005; appeared online Feb. 18, 2005; published online May 11, 2005.

\section{Introduction}

In many applications of fiber optics, such as optical communications and optical measurements, it is desirable to have fiber-pigtailed broadband light sources with flat spectra and high power output. ${ }^{1,2}$ Various light-emitting diodes such as superluminescence LED (SLED) are usually used as the light sources. However, the output spectra of LEDs are normally Gaussian-like. Therefore, it is of practical importance to develop an equalization filter to equalize Gaussian-like spectra. The filter should have two critical specifications. First, it should be able to equalize the spectrum to make it as flat as possible. Second, it should have as minimal an attenuation as possible in order to obtain the maximum power output.
Over the last few decades, much of the focus on spectrum equalization has been given to the problem of gain equalization of erbium-doped fiber amplifiers (EDFAs). ${ }^{3}$ However, the existing equalization filters developed for EDFAs are not suitable for broadband light sources since they do not address the issue of minimal attenuation. Recently, a fiber equalizer for equalizing Gaussian-like spectra was developed using a $2 \times 2$ fiber lattice. ${ }^{4}$ But substantial attenuation is introduced because a certain amount of input power is lost through the second output port of the filter.

In this paper, we propose a new fiber equalization filter, which consists of symmetric forward and feedback lattices. We show that the proposed filter can achieve a high power output with the desired flat output spectrum. The symmetric feedback structure also enables an analytic design of the filter. Using the proposed filter, the equalization of a SLED spectrum is demonstrated with $0.1-\mathrm{dB}$ ripple over $30 \mathrm{~nm}$ of bandwidth. Compared to existing results, ${ }^{4}$ the filter has lower attenuation while attaining the same flatness of the output spectrum.

\section{Equalization Filter with Symmetric Forward and Feedback Lattice Structure}

The proposed spectrum equalizer consists of two fiber lattice filters, denoted respectively by forward lattice and feedback lattice, as shown in Fig. 1. Each lattice filter is a fiber Mach-Zehnder interferometer formed by cascading three fiber couplers with differential time delay lines. The two lattice filters are designed to be the same, but they are connected into a loop in a reverse manner. Using this fiber filter as an equalizer, it accepts the input light with a Gaussian-like spectrum at input port 1 or port 2 as shown in Fig. 1, and outputs the equalized spectrum at output port 3 or port 4 , correspondingly.

To illustrate the design of the equalizer, we introduce the optical frequency as $w=\Delta L \pi n / 2 \lambda$ where $\lambda$ is the wavelength, $n$ is the refractive index of the optical fiber, and all the differential lengths are chosen identically to be $\Delta L$. Thus, the input Gaussian-like spectrum is expressed by $F(w)$. For the forward lattice without the feedback lattice connected into the loop, the bar-state power transmission from port 1 to port 3 is denoted as $P_{b a r}(w)$. The closedloop bar-state power transmission from port 1 to port 3 is denoted as $P(w)$. Applying the equalization filter to the spectrum $F(w)$, the equalized spectrum is expressed by $P(w) F(w)$. The objective of the equalizer design can be described as one of designing all-fiber coupling ratios such that the output spectrum $P(w) F(w)$ is as flat as possible in the passband and its central power is as high as possible.

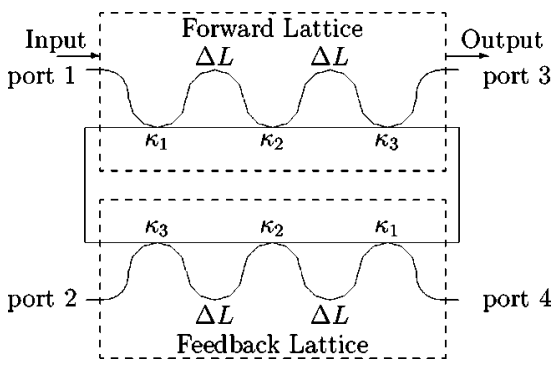

Fig. 1 Proposed equalization filter with feedback structure. 


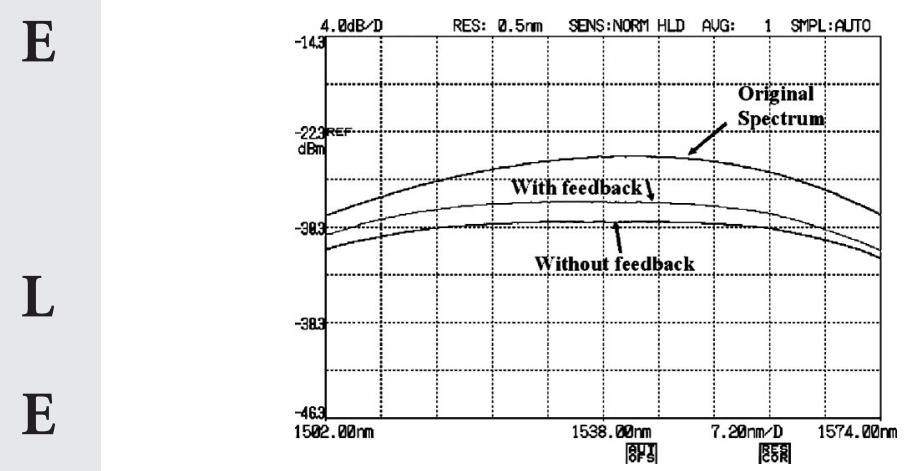

$\mathrm{T}$

$\mathrm{T}$

E

$\mathbf{R}$ transmission.
Fig. 2 Experimental SLED spectrum responses with maximally flat

The design of such an equalizer can be done in two steps. In the first step, an analytic Gaussian function is used to approximate the original Gaussian-like spectrum. The Gaussian function used is expressed as $F(w)$ $=\beta \cdot \exp \left[-\left(w-w_{0}\right)^{2} / 2 \delta^{2}\right]$, where $w_{0}$ corresponds to the central wavelength, $\beta$ the amplitude, and $\delta$ the deviation of the Gaussian function. The approximation can be done in a least-squares sense when the measurement of the original Gaussian-like spectrum is available. In the second step, an equalization filter with a transmission spectrum denoted by $P(w)$ is designed. From the field transmission analysis, the closed-loop bar-state power transmission $P(w)$ is expressed as $P(w)=\left[2 P_{b a r}(w)\right] /\left[1+P_{b a r}(w)\right]$ by using the symmetric lattice structure of the filter. The desired fiber coupling ratios can be determined by optimizing the output power at the central wavelength $w=w_{0}$ with constraints that the output spectrum is as flat as possible in the neighborhood of the central wavelength. Constraints on flatness can be obtained by applying the maximally flat conditions of an analytic function to $P(w) F(w)$ at $w=w_{0}{ }^{4}$

As an illustrative example, we consider the equalization of a Gaussian-like spectrum as used in the subsequent experiments. The spectrum is given in Fig. 2. Using a leastsquares method, the spectrum can be approximated by choosing $\delta=0.66$ and $w_{0}=1538 \mathrm{~nm}$. Thus, we take $\Delta L$ $=18.3 \mu \mathrm{m}$ so that the central wavelength of the equalization filter sits at $1538 \mathrm{~nm}$. For this case, it is easy to verify that it suffices to evaluate the first- and third-order derivatives of $P_{b a r}(w) F(w)$ with respect to $w$ at $w=w_{0}$. The obtained optimal coupling ratios are $\kappa_{1}=0.52 \%, \kappa_{2}$ $=75.89 \%$, and $\kappa_{3}=33 \%$ for the first, second, and third fiber couplers in the forward lattice.

\section{Experimental Results}

To demonstrate the effectiveness of the proposed filter, we build it in experiments to equalize the SLED spectrum. As shown in the previous section, the desired coupling ratios are obtained as $\kappa_{1}=0.52 \%, \kappa_{2}=75.89 \%$, and $\kappa_{3}=33 \%$. Using the fabrication technique, ${ }^{5}$ the two lattice filters are made separately by fabricating three fiber couplers successively on two piece fibers. This fabrication technique does not require any splicing so it eliminates the possible splicing loss. The first coupler is fabricated by a fiber coupling fusion workstation with the coupling ratio set at $0.52 \%$. The second and the third couplers are fabricated with the help of a broadband light source and an optical spectrum analyzer (OSA). Since the isolation of the interference pattern is determined only by the coupling ratios of the two couplers, and the period of the interference pattern depends on the length difference between the two couplers, the splitting ratio of the second coupler can be estimated by observing that the interference pattern reaches the expected intensity contrast. When fusing the second and the third couplers, the fiber lengths between the couplers are made roughly the same. After fabrication of the second and third couplers, one of the two fibers is then stretched to achieve the desired length difference by monitoring the interference pattern on the OSA.

Connecting the filter to the output of the SLED where the Gaussian-like spectrum is measured, the equalized spectrum is observed on the OSA, as shown in Fig. 2. For the purpose of demonstrating the effectiveness of the proposed method, an equalization filter without feedback structure $^{4}$ is also fabricated. The flattened spectra are shown in Fig. 2 for comparison. We show that the proposed equalization filter can equalize the SLED spectrum with $0.1-\mathrm{dB}$ ripples over a $30-\mathrm{nm}$ bandwidth. This is the same as the results of using the equalization filter without a feedback lattice. However, the output power in the 0.1-dB passband is increased by $2 \mathrm{~dB}$ per wavelength. This demonstrates the effectiveness of the proposed design approach.

We have done simulations and experiments by using a higher number of taps in the fiber lattices. The results have shown that when more taps are used, the equalized spectrum is flatter but the output power is lower. Therefore, a compromise between the flatness and the output power must be taken into consideration in practice, particularly in the presence of coupling losses due to imperfect fabrication of the fiber couplers.

\section{Conclusion}

This paper presents a new all-fiber lattice filter to equalize Gaussian-like spectrums. Compared to existing filters for the same purpose, the proposed equalized filter can yield higher throughput. In addition, the symmetry between the forward and feedback lattices enables an analytic design. This has greatly simplified the task of filter design. Experimental results have demonstrated that the proposed filter can flatten the spectrum of a SLED with the same performance of flatness as that of existing results, and the benefit is that the attenuation is reduced by $2 \mathrm{~dB}$.

\section{Acknowledgment}

This paper is supported by SIMTech U04-P-106B.

\section{References}

1. J. Gowar, Optical Communications Systems, 2nd ed., Prentice-Hall, Englewood Cliffs, NJ (1993).

2. G. Keiser, Optical Fibre Communications, 3rd ed., McGraw-Hill, New York (2000).

3. P. F. Wysocki, "Broad-band erbium-doped fiber amplifier flattened beyond $40 \mathrm{~nm}$ using long-period grating filter," IEEE Photonics Technol. Lett. 9, 1343-1345 (1997).

4. Y. Zhang, Q. J. Wang, and Y. C. Soh, "Equalization of Gaussianlikespectra with optical lattice filters," J. Opt. Soc. Am. B (to be published).

5. Q. J. Wang, T. Liu, Y. C. Soh, and Y. Zhang, "All-fiber $\mathrm{F}^{3} \mathrm{~T}$ interleaver design with specified performance parameters," Opt. Eng. 42(11), 3172-3178 (2003). 